\title{
Dynamics of a Stochastic Delayed Competitive Model with Impulsive Toxicant Input in Polluted Environments
}

\author{
Meiling Deng, Meng Liu, and Chuanzhi Bai \\ School of Mathematical Science, Huaiyin Normal University, Huaian 223300, China \\ Correspondence should be addressed to Chuanzhi Bai; czbai@hytc.edu.cn \\ Received 1 March 2014; Revised 30 April 2014; Accepted 4 May 2014; Published 25 May 2014 \\ Academic Editor: Rehana Naz
}

Copyright (C) 2014 Meiling Deng et al. This is an open access article distributed under the Creative Commons Attribution License, which permits unrestricted use, distribution, and reproduction in any medium, provided the original work is properly cited.

In the world today, with the rapid development of modern agriculture and industry, a large quantity of pollutants enter into ecosystems one by one, which is a threat to the persistence of the exposed populations. This paper investigates a stochastic delayed competitive system with impulsive toxicant input in a polluted environment. Under a simple condition, sufficient and necessary conditions for stability in the mean and extinction of each species are established. Some recent works are improved and extended greatly. Some numerical simulations are also included to illustrate and support the findings.

\section{Introduction}

In this paper, we consider the following stochastic delay competitive model in polluted environments with impulsive toxicant input:

$$
\begin{aligned}
& d N_{1}(t)=N_{1}(t)\left[r_{10}-r_{11} C_{0}(t)-a_{11} N_{1}(t)\right. \\
& \left.-a_{12} N_{2}\left(t-\tau_{1}\right)\right] d t+\lambda_{1} N_{1}(t) d W_{1}(t), \\
& d N_{2}(t)=N_{2}(t)\left[r_{20}-r_{21} C_{0}(t)-a_{22} N_{2}(t)\right. \\
& \left.-a_{21} N_{1}\left(t-\tau_{2}\right)\right] d t+\lambda_{2} N_{2}(t) d W_{2}(t), \\
& \frac{d C_{0}(t)}{d t}=k C_{e}(t)-(g+m) C_{0}(t) \text {, } \\
& \frac{d C_{e}(t)}{d t}=-h C_{e}(t) \text {, } \\
& t \neq n \gamma, \quad n \in Z^{+} \\
& \Delta N_{i}(t)=0, \quad \Delta C_{0}(t)=0, \quad \Delta C_{e}(t)=b, \\
& t=n \gamma, \quad n \in Z^{+}, \quad i=1,2,
\end{aligned}
$$

with initial data

$$
\begin{array}{r}
N_{i}(t)=\psi_{i}(t)>0, \\
t \in[-\tau, 0] ; \quad \psi_{i}(0)>0, \quad i=1,2,
\end{array}
$$

where $\tau_{i} \geq 0, \tau=\max \left\{\tau_{1}, \tau_{2}\right\}$, and $\psi_{i}(t)$ is a continuous function on $[-\tau, 0]$. All coefficients in model (1) are positive. $\Delta f(t)=f\left(t^{+}\right)-f(t)$ and $Z^{+}=\{1,2, \ldots\} ; N_{i}(t)$ is the size of the $i$ th population, $i=1,2 ; r_{i 0}$ is the growth rate of the $i$ th population; $r_{i 1}$ is the response to the pollutant present in the organism of the $i$ th population; $C_{0}(t)$ is the toxicant concentration in the organism; $C_{e}(t)$ is the toxicant concentration in the environment; $k C_{e}(t)$ is the organism's net uptake of toxicant from the environment; $g C_{0}(t)+m C_{0}(t)$ is the egestion and depuration rates of the toxicant in the organism; $h C_{e}(t)$ is the toxicant loss from the environment itself; $\gamma$ is the period of the impulsive effect about the exogenous input of toxicant; $b$ is the amount of toxicant input at every time. $W_{i}(t)$ is a standard Brownian motion defined on a complete probability space $(\Omega, \mathscr{F}, \mathscr{P}) ; \lambda_{i}^{2}$ is the intensity of the environmental noise.

Recently, population models with toxicant effect have received great attention; see, for example, [1-18]. Liu and 
Zhang [15] considered the following competitive model in polluted environments with impulsive toxicant input:

$$
\begin{aligned}
& \frac{d N_{1}(t)}{d t} \\
& \quad=N_{1}(t)\left[r_{10}-r_{11} C_{0}(t)-a_{11} N_{1}(t)-a_{12} N_{2}(t)\right], \\
& \frac{d N_{2}(t)}{d t} \\
& =N_{2}(t)\left[r_{20}-r_{21} C_{0}(t)-a_{21} N_{1}(t)-a_{22} N_{2}(t)\right], \\
& \frac{d C_{0}(t)}{d t}=k C_{e}(t)-(g+m) C_{0}(t), \\
& \frac{d C_{e}(t)}{d t}=-h C_{e}(t), \quad t \neq n \gamma, \quad n \in Z^{+} . \\
& \Delta N_{i}(t)=0, \quad \Delta C_{0}(t)=0, \quad \Delta C_{e}(t)=b, \\
& \quad t=n \gamma, \quad n \in Z^{+}, \quad i=1,2,
\end{aligned}
$$

For model (3), the authors [15] proved the following.

Lemma 1 (see [15]). Define

$$
\begin{array}{ll}
\Gamma=a_{11} a_{22}-a_{12} a_{21}, & \Gamma_{1}=r_{10} a_{22}-a_{12} r_{20}, \\
\Gamma_{2}=r_{20} a_{11}-r_{10} a_{21}, & \bar{\Gamma}_{1}:=a_{22} r_{11}-a_{12} r_{21}, \\
\bar{\Gamma}_{2}:=a_{11} r_{21}-a_{21} r_{11}, & K=\frac{k b}{\gamma h(g+m)} .
\end{array}
$$

Suppose that $\Gamma>0, \Gamma_{1}>0, \Gamma_{2}>0$.

(A) If $\Gamma_{1}>\bar{\Gamma}_{1} K$ and $\Gamma_{2}<\bar{\Gamma}_{2} K$, then $\lim _{t \rightarrow+\infty} N_{2}(t)=0$ and $\lim \sup _{t \rightarrow+\infty} t^{-1} \int_{0}^{t} N_{1}(s) d s>0$.

(B) If $\Gamma_{1}<\bar{\Gamma}_{1} K$ and $\Gamma_{2}>\bar{\Gamma}_{2} K$, then $\lim _{t \rightarrow+\infty} N_{1}(t)=0$ and $\lim \sup _{t \rightarrow+\infty} t^{-1} \int_{0}^{t} N_{2}(s) d s>0$.

(C) If $\Gamma_{1}>\bar{\Gamma}_{1} K$ and $\Gamma_{2}>\bar{\Gamma}_{2} K$, then $\lim \sup _{t \rightarrow+\infty} t^{-1} \int_{0}^{t} N_{i}(s) d s>0, i=1,2$.

From the work of Liu and Zhang [15], some important and interesting questions arise naturally.

(Q1) In the real world, the growth of population is inevitably affected by random environmental fluctuations. May [19] have claimed that population systems should be stochastic. Therefore, what happens if system (3) is affected by environmental fluctuations?

(Q2) Gopalsamy [20] have pointed out that, in order to be reality, time delays should not be ignored. Hence, what happens if system (3) incorporates with time delays?

(Q3) Can we improve the results obtained in Lemma 1?

The aims of this paper are to investigate the above questions. Recall that $r_{i 0}$ stands for the growth rate. In practice, we often estimate it by an average value plus an error term. Generally, by the famous central limit theorem, the error term follows a normal distribution. Hence, for short correlation time, we can replace $r_{i 0}$ with $r_{i 0}+\lambda_{i} \dot{W}_{i}(t)$ (see, e.g., [21-29]), where $\dot{W}_{i}(t)$ is white noise and $\lambda_{i}^{2}$ is the intensity of the noise. At the same time, incorporating with time delays, we get model (1). For model (1), we will show the following.

Theorem 2. Suppose that $\Gamma>0$. Define

$$
\begin{aligned}
& \eta_{i}=r_{i 0}-r_{i 1} K-0.5 \lambda_{i}^{2}, \quad i=1,2, \\
& \widetilde{\Gamma}_{1}=0.5 a_{22} \lambda_{1}^{2}-0.5 a_{12} \lambda_{2}^{2}, \quad \widetilde{\Gamma}_{2}=0.5 a_{11} \lambda_{2}^{2}-0.5 a_{21} \lambda_{1}^{2} .
\end{aligned}
$$

(I) If $\eta_{1}<0$ and $\eta_{2}<0$, then both $N_{1}$ and $N_{2}$ go to extinction almost surely; that is,

$$
\lim _{t \rightarrow+\infty} N_{i}(t)=0 \quad \text { a.s., } i=1,2
$$

(II) if $\eta_{1}>0$ and $\eta_{2}<0$, then $N_{2}$ goes to extinction a.s. and $N_{1}$ is stable in the mean a.s.; that is,

$$
\lim _{t \rightarrow+\infty} t^{-1} \int_{0}^{t} N_{1}(s) d s=\frac{\eta_{1}}{a_{11}}, \quad \text { a.s.; }
$$

(III) if $\eta_{1}<0$ and $\eta_{2}>0$, then $N_{1}$ goes to extinction a.s. and $\mathrm{N}_{2}$ is stable in the mean a.s.; that is,

$$
\lim _{t \rightarrow+\infty} t^{-1} \int_{0}^{t} N_{2}(s) d s=\frac{\eta_{2}}{a_{22}}, \quad \text { a.s.; }
$$

(IV) if $\eta_{1}>0, \eta_{2}>0$,

(A) if $\Gamma_{1}>\bar{\Gamma}_{1} K+\widetilde{\Gamma}_{1}$ and $\Gamma_{2}<\bar{\Gamma}_{2} K+\widetilde{\Gamma}_{2}$, then $N_{2}$ goes to extinction a.s. and $N_{1}$ is stable in the mean a.s.

$$
\lim _{t \rightarrow+\infty} t^{-1} \int_{0}^{t} N_{1}(s) d s=\frac{\eta_{1}}{a_{11}}, \quad \text { a.s.; }
$$

(B) if $\Gamma_{1}<\bar{\Gamma}_{1} K+\widetilde{\Gamma}_{1}$ and $\Gamma_{2}>\bar{\Gamma}_{2} K+\widetilde{\Gamma}_{2}$, then $N_{1}$ goes to extinction a.s. and $\mathrm{N}_{2}$ is stable in the mean a.s.

$$
\lim _{t \rightarrow+\infty} t^{-1} \int_{0}^{t} N_{2}(s) d s=\frac{\eta_{2}}{a_{22}}, \quad \text { a.s.; }
$$

(C) if $\Gamma_{1}>\bar{\Gamma}_{1} K+\widetilde{\Gamma}_{1}$ and $\Gamma_{2}>\bar{\Gamma}_{2} K+\widetilde{\Gamma}_{2}$, then both $N_{1}$ and $\mathrm{N}_{2}$ are stable in the mean a.s.

$$
\begin{aligned}
& \lim _{t \rightarrow+\infty} t^{-1} \int_{0}^{t} N_{1}(s) d s=\frac{\Gamma_{1}-\bar{\Gamma}_{1} K-\widetilde{\Gamma}_{1}}{\Gamma}, \quad \text { a.s. } \\
& \lim _{t \rightarrow+\infty} t^{-1} \int_{0}^{t} N_{2}(s) d s=\frac{\Gamma_{2}-\bar{\Gamma}_{1} K-\widetilde{\Gamma}_{2}}{\Gamma}, \quad \text { a.s. }
\end{aligned}
$$

Remark 3. It is useful to point out that if $\eta_{1}>0, \eta_{2}>0$, and $\Gamma>0$, then $\Gamma_{1}<\bar{\Gamma}_{1}+\widetilde{\Gamma}_{1}$ and $\Gamma_{2}<\bar{\Gamma}_{2}+\widetilde{\Gamma}_{2}$ will not hold simultaneously. 
Remark 4. In comparison with most of the existing results, our key contributions in this paper are as follows.

(i) To the best of our knowledge, this paper is the first attempt to consider stochastic delay competitive model in polluted environments.

(ii) Our conditions are much weaker. For example, the authors [15] supposed $\Gamma_{1}>0$ and $\Gamma_{2}>0$ which are dropped in this paper.

(iii) Our results improve some recent works. For example, Lemma 1 indicates that the superior limit is positive while Theorem 2 proves that the limit exists and establishes the explicit form of the limit.

\section{Proof}

For simplicity, define

$$
\begin{gathered}
R_{+}^{2}=\left\{a=\left(a_{1}, a_{2}\right) \in R^{2} \mid a_{i}>0, i=1,2\right\}, \\
\langle g(t)\rangle=t^{-1} \int_{0}^{t} g(s) d s .
\end{gathered}
$$

Lemma 5. For any given initial data $\psi(\theta)=\left(\psi_{1}(\theta), \psi_{2}(\theta)\right) \epsilon$ $C\left([-\tau, 0], R_{+}^{2}\right)$, there is a unique global positive solution $N(t)=$ $\left(N_{1}(t), N_{2}(t)\right)$ to model (1) on $t \geq-\tau$ a.s. and

$$
\limsup _{t \rightarrow+\infty} \frac{\ln N_{i}(t)}{\ln t} \leq 1 \quad \text { a.s., } i=1,2 .
$$

Proof. The proof is a special case of Theorems 5.1 and 5.2 in Liu and Wang [25] and hence is omitted.

Lemma 6 (see [26]). Suppose that $x(t) \in C\left[\Omega \times[0,+\infty), R_{+}\right]$.

(I) If there exist $\delta$ and positive constants $\delta_{0}$, T such that

$$
\ln x(t) \leq \delta t-\delta_{0} \int_{0}^{t} x(s) d s+\sum_{i=1}^{n} \beta_{i} W_{i}(t)
$$

for $t \geq T$, where $W_{i}(t)$ are independent standard Brownian motions and $\beta_{i}$ are constants, $1 \leq i \leq$ $n$, then we have the following: if $\delta \geq 0$, then $\limsup \sup _{t \rightarrow+\infty}\langle x(t)\rangle \leq \delta / \delta_{0}$ a.s.; if $\delta<0$, then $\lim _{t \rightarrow+\infty} x(t)=0$ a.s.

(II) If there exist positive constants $\delta_{0}, T$, and $\delta$ such that

$$
\ln x(t) \geq \delta t-\delta_{0} \int_{0}^{t} x(s) d s+\sum_{i=1}^{n} \beta_{i} W_{i}(t)
$$

for $t \geq T$, then $\liminf _{t \rightarrow+\infty}\langle x(t)\rangle \geq \delta / \delta_{0}$ a.s. (1):

To begin with, let us consider the following subsystem of

$$
\begin{array}{r}
\frac{d C_{0}(t)}{d t}=k C_{e}(t)-(g+m) C_{0}(t), \\
\frac{d C_{e}(t)}{d t}=-h C_{e}(t), \\
t \neq n \gamma, n \in Z^{+}, \\
\Delta C_{0}(t)=0, \quad \Delta C_{e}(t)=b, \quad t=n \gamma, n \in Z^{+} \\
0 \leq C_{0}(0) \leq 1,0 \leq C_{e}(0) \leq 1 .
\end{array}
$$

Lemma 7 (see $[12,13]$ ). System (16) has a unique positive $\gamma$-periodic solution $\left(\widetilde{C}_{0}(t), \widetilde{C}_{e}(t)\right)^{T}$ and for each solution $\left(C_{0}(t), C_{e}(t)\right)^{T}$ of $(16), C_{0}(t) \rightarrow \widetilde{C}_{0}(t)$ and $C_{e}(t) \rightarrow \widetilde{C}_{e}(t)$ as $t \rightarrow \infty$. Moreover, $C_{0}(t)>\widetilde{C}_{0}(t)$ and $C_{e}(t)>\widetilde{C}_{e}(t)$ for all $t \geq 0$ if $C_{0}(0)>\widetilde{C}_{0}(0)$ and $C_{e}(0)>\widetilde{C}_{e}(0), i=1,2$, where

$$
\begin{aligned}
& \widetilde{C}_{0}(t)=\widetilde{C}_{0}(0) e^{-(g+m)(t-n \gamma)}+\frac{k b\left(e^{-(g+m)(t-n \gamma)}-e^{-h(t-n \gamma)}\right)}{(h-g-m)\left(1-e^{-h \gamma}\right)}, \\
& \widetilde{C}_{e}(t)=\frac{b e^{-h(t-n \gamma)}}{1-e^{-h \gamma}}, \\
& \widetilde{C}_{0}(0)=\frac{k b\left(e^{-(g+m) \gamma}-e^{-h \gamma}\right)}{(h-g-m)\left(1-e^{-(g+m) \gamma}\right)\left(1-e^{-h \gamma}\right)}, \\
& \widetilde{C}_{e}(0)=\frac{b}{1-e^{-h \gamma}}
\end{aligned}
$$

for $t \in(n \gamma,(n+1) \gamma]$ and $n \in Z^{+}$. In addition,

$$
\lim _{t \rightarrow+\infty} t^{-1} \int_{0}^{t} \widetilde{C}_{0}(s) d s=\frac{k b}{h(g+m) \gamma}=K .
$$

Proof of Theorem 2. It follows from Lemma 7 that

$$
\lim _{t \rightarrow+\infty} t^{-1} \int_{0}^{t} C_{0}(s) d s=\lim _{t \rightarrow+\infty} t^{-1} \int_{0}^{t} \widetilde{C}_{0}(s) d s=K .
$$

That is to say, for all $\varepsilon>0$, there is $T>0$ such that

$$
K-\varepsilon \leq\left\langle C_{0}(t)\right\rangle \leq K+\varepsilon, \quad t>T
$$

Applying Itô's formula to (1) leads to

$d \ln N_{1}(t)$

$$
\begin{aligned}
= & \frac{d N_{1}(t)}{N_{1}(t)}-\frac{\left(d N_{1}(t)\right)^{2}}{2 N_{1}^{2}(t)} \\
= & {\left[r_{10}-0.5 \lambda_{1}^{2}-r_{11} C_{0}(t)-a_{11} N_{1}(t)-a_{12} N_{2}\left(t-\tau_{1}\right)\right] d t } \\
& +\lambda_{1} d W_{1}(t)
\end{aligned}
$$

$d \ln N_{2}(t)$

$$
\begin{aligned}
= & \frac{d N_{2}(t)}{N_{2}(t)}-\frac{\left(d N_{2}(t)\right)^{2}}{2 N_{2}^{2}(t)} \\
= & {\left[r_{20}-0.5 \lambda_{2}^{2}-r_{21} C_{0}(t)-a_{21} N_{1}\left(t-\tau_{2}\right)-a_{22} N_{2}(t)\right] d t } \\
& +\lambda_{2} d W_{2}(t) .
\end{aligned}
$$


In other words, we have shown that

$$
\begin{aligned}
\ln N_{1}( & t)-\ln N_{1}(0) \\
= & \left(r_{10}-0.5 \lambda_{1}^{2}\right) t-r_{11} \int_{0}^{t} C_{0}(s) d s-a_{11} \int_{0}^{t} N_{1}(s) d s \\
& -a_{12} \int_{0}^{t} N_{2}\left(s-\tau_{1}\right) d s+\lambda_{1} W_{1}(t) \\
= & \left(r_{10}-0.5 \lambda_{1}^{2}\right) t-a_{12} \int_{0}^{t} N_{2}(s) d s \\
& +a_{12}\left[\int_{t-\tau_{1}}^{t} N_{2}(s) d s-\int_{-\tau_{1}}^{0} N_{2}(s) d s\right] \\
& -r_{11} \int_{0}^{t} C_{0}(s) d s-a_{11} \int_{0}^{t} N_{1}(s) d s+\lambda_{1} W_{1}(t),
\end{aligned}
$$

$\ln N_{2}(t)-\ln N_{2}(0)$

$$
\begin{aligned}
= & \left(r_{20}-0.5 \lambda_{2}^{2}\right) t-r_{21} \int_{0}^{t} C_{0}(s) d s \\
& -a_{21} \int_{0}^{t} N_{1}\left(s-\tau_{2}\right) d s-a_{22} \int_{0}^{t} N_{2}(s) d s+\lambda_{2} W_{2}(t) \\
= & \left(r_{20}-0.5 \lambda_{2}^{2}\right) t-a_{21} \int_{0}^{t} N_{1}(s) d s \\
& +a_{21}\left[\int_{t-\tau_{2}}^{t} N_{1}(s) d s-\int_{-\tau_{2}}^{0} N_{1}(s) d s\right] \\
& -r_{21} \int_{0}^{t} C_{0}(s) d s-a_{22} \int_{0}^{t} N_{2}(s) d s+\lambda_{2} W_{2}(t) .
\end{aligned}
$$

(I) Assume that $\eta_{1}=r_{10}-0.5 \lambda_{1}^{2}-r_{11} K<0$ and $\eta_{2}=$ $r_{20}-0.5 \lambda_{2}^{2}-r_{21} K<0$. In view of $(22)$,

$$
t^{-1} \ln \frac{N_{1}(t)}{N_{1}(0)} \leq r_{10}-r_{11} \int_{0}^{t} C_{0}(s) d s-0.5 \lambda_{1}^{2}+t^{-1} \lambda_{1} W_{1}(t)
$$

By $\lim _{t \rightarrow+\infty} W_{i}(t) / t=0$ a.s., $i=1,2(19)$ and $r_{10}<0.5 \lambda_{1}^{2}+$ $r_{11} K$ we have that

$$
\limsup _{t \rightarrow+\infty} t^{-1} \ln N_{1}(t) \leq r_{10}-0.5 \lambda_{1}^{2}-r_{11} K=\eta_{1}<0 .
$$

Therefore, $\lim _{t \rightarrow+\infty} N_{1}(t)=0$, a.s. Similarly, it follows from (9) that if $\eta_{2}<0$, then $\lim _{t \rightarrow+\infty} N_{2}(t)=0$, a.s.

(II) Assume that $\eta_{1}>0$ and $\eta_{2}<0$. Since $\eta_{2}<0$, then, by (I), $\lim _{t \rightarrow+\infty} N_{2}(t)=0$, a.s. Hence, for arbitrary $\varepsilon>0$, there exists $T>0$ such that for $t \geq T$

$$
\begin{aligned}
-\frac{\varepsilon}{2} & \leq a_{12} t^{-1} \int_{0}^{t} N_{2}\left(s-\tau_{1}\right) d s \leq \frac{\varepsilon}{2}, \\
-\frac{\varepsilon}{2} & \leq t^{-1} \ln N_{1}(0) \leq \frac{\varepsilon}{2} .
\end{aligned}
$$

When the above inequalities and (20) are used in (22), we can obtain that, for $t \geq T$,

$$
\begin{aligned}
& \ln N_{1}(t) \leq\left(\eta_{1}+2 \varepsilon\right) t-a_{11} \int_{0}^{t} N_{1}(s) d s+\lambda_{1} W_{1}(t), \\
& \ln N_{1}(t) \geq\left(\eta_{1}-2 \varepsilon\right) t-a_{11} \int_{0}^{t} N_{1}(s) d s+\lambda_{1} W_{1}(t) .
\end{aligned}
$$

Note that $\eta_{1}>0$; we can let $\varepsilon$ be sufficiently small such that $\eta_{1}-2 \varepsilon>0$. Applying (i) and (ii) in Lemma 6 to (26) and (27), respectively, one can see that

$$
\begin{aligned}
\frac{\eta_{1}-2 \varepsilon}{a_{11}} & \leq \liminf _{t \rightarrow+\infty}\left\langle N_{1}(t)\right\rangle \leq \limsup _{t \rightarrow+\infty}\left\langle N_{1}(t)\right\rangle \\
& \leq \frac{\eta_{1}+2 \varepsilon}{a_{11}}, \quad \text { a.s. }
\end{aligned}
$$

It therefore follows from the arbitrariness of $\varepsilon$ that

$$
\lim _{t \rightarrow+\infty}\left\langle N_{1}(t)\right\rangle=\frac{\eta_{1}}{a_{11}}, \quad \text { a.s. }
$$

The proof of (III) can be obtained similarly and hence is omitted.

Now, we are in the position to prove (IV). Assume that $\eta_{1}>0$ and $\eta_{2}>0$. For $i=1,2$, consider the following stochastic equation:

$$
\begin{gathered}
d y_{i}(t)=y_{i}(t)\left[r_{i 0}-r_{i 1} C_{0}(t)-a_{i i} y_{i}(t)\right] d t+\lambda_{i} y_{i}(t) d W_{i}(t), \\
y_{i}(\theta)=\psi(\theta), \quad \theta \in[-\tau, 0] .
\end{gathered}
$$

By the classical stochastic comparison theorem [30], one can see that

$$
N_{1}(t) \leq y_{1}(t), \quad N_{2}(t) \leq y_{2}(t) .
$$

Note that $r_{i 0}>0.5 \lambda_{i}^{2}+r_{i 1} K, i=1,2$; an argument, identical to the argument used in the proof of (II), shows that

$$
\lim _{t \rightarrow+\infty}\left\langle y_{i}(t)\right\rangle=\lim _{t \rightarrow+\infty} t^{-1} \int_{0}^{t} y_{i}(s) d s=\frac{\eta_{i}}{a_{i i}} \quad \text { a.s., } i=1,2 .
$$

Consequently,

$$
\begin{aligned}
& \lim _{t \rightarrow+\infty} t^{-1} \int_{t-\tau_{1}}^{t} y_{2}(s) d s \\
& =\lim _{t \rightarrow+\infty}\left(t^{-1} \int_{0}^{t} y_{2}(s) d s-t^{-1} \int_{0}^{t-\tau_{1}} y_{2}(s) d s\right)=0 \\
& \lim _{t \rightarrow+\infty} t^{-1} \int_{t-\tau_{2}}^{t} y_{1}(s) d s=0, \quad \text { a.s. }
\end{aligned}
$$

This, together with (31), implies that

$$
\begin{gathered}
\lim _{t \rightarrow+\infty} t^{-1} \int_{t-\tau_{1}}^{t} N_{2}(s) d s=0, \\
\lim _{t \rightarrow+\infty} t^{-1} \int_{t-\tau_{2}}^{t} N_{1}(s) d s=0, \quad \text { a.s. }
\end{gathered}
$$


On the other hand, computing $(9) \times a_{11}-(22) \times a_{21}$ gives

$$
\begin{aligned}
a_{11} \ln \frac{N_{2}(t)}{N_{2}(0)} & =a_{11} a_{21}\left[\int_{t-\tau_{2}}^{t} N_{1}(s) d s-\int_{-\tau_{2}}^{0} N_{1}(s) d s\right] \\
& -a_{21} a_{12}\left[\int_{t-\tau_{1}}^{t} N_{2}(s) d s-\int_{-\tau_{1}}^{0} N_{2}(s) d s\right] \\
& +a_{21} \ln \frac{N_{1}(t)}{N_{1}(0)}+\left(\Gamma_{2}-\widetilde{\Gamma}_{2}\right) t-\bar{\Gamma}_{2} \int_{0}^{t} C_{0}(s) d s \\
& -\Gamma \int_{0}^{t} N_{2}(s) d s-a_{21} \lambda_{1} W_{1}(t)+a_{11} \lambda_{2} W_{2}(t) .
\end{aligned}
$$

In view of (13) and (34), for arbitrary $\varepsilon>0$, there exits $T>0$ such that, for $t \geq T$,

$$
\begin{gathered}
t^{-1} a_{21} \ln \frac{N_{1}(t)}{N_{1}(0)}<\frac{\varepsilon}{3}, \quad t^{-1} a_{11} \ln N_{2}(0)<\frac{\varepsilon}{3}, \\
t^{-1} a_{11} a_{21}\left[\int_{t-\tau_{2}}^{t} N_{1}(s) d s-\int_{-\tau_{2}}^{0} N_{1}(s) d s\right] \\
-t^{-1} a_{21} a_{12}\left[\int_{t-\tau_{1}}^{t} N_{2}(s) d s-\int_{-\tau_{1}}^{0} N_{2}(s) d s\right] \leq \frac{\varepsilon}{3} .
\end{gathered}
$$

When the above inequalities and (20) are used in (35), we can see that for $t>T$

$$
\begin{aligned}
a_{11} \ln & N_{2}(t) \\
\leq & \left(\Gamma_{2}-\bar{\Gamma}_{2} K-\widetilde{\Gamma}_{2}+2 \varepsilon\right) t \\
& \quad-\Gamma \int_{0}^{t} N_{2}(s) d s-a_{21} \lambda_{1} W_{1}(t)+a_{11} \lambda_{2} W_{2}(t) .
\end{aligned}
$$

Similarly, computing $(22) \times a_{22}-(9) \times a_{12}$ gives

$$
\begin{aligned}
a_{22} \ln \frac{N_{1}(t)}{N_{1}(0)} & \\
= & a_{22} a_{12}\left[\int_{t-\tau_{1}}^{t} N_{2}(s) d s-\int_{-\tau_{1}}^{0} N_{2}(s) d s\right] \\
& -a_{12} a_{21}\left[\int_{t-\tau_{2}}^{t} N_{1}(s) d s-\int_{-\tau_{2}}^{0} N_{1}(s) d s\right] \\
& +a_{12} \ln \frac{N_{2}(t)}{N_{2}(0)}+\left(\Gamma_{1}-\widetilde{\Gamma}_{1}\right) t-\bar{\Gamma}_{1} \int_{0}^{t} C_{0}(s) d s \\
& -\Gamma \int_{0}^{t} N_{1}(s) d s+a_{22} \lambda_{1} W_{1}(t)-a_{12} \lambda_{2} W_{2}(t) .
\end{aligned}
$$

Hence, for sufficiently large $t$,

$$
\begin{aligned}
a_{22} \ln & N_{1}(t) \\
\leq & \left(\Gamma_{1}-\bar{\Gamma}_{1} K-\widetilde{\Gamma}_{1}+2 \varepsilon\right) t \\
& \quad-\Gamma \int_{0}^{t} N_{1}(s) d s+a_{22} \lambda_{1} W_{1}(t)-a_{12} \lambda_{2} W_{2}(t) .
\end{aligned}
$$

(A) Assume that $\Gamma_{1}>\bar{\Gamma}_{1} K+\widetilde{\Gamma}_{1}$ and $\Gamma_{2}<\bar{\Gamma}_{2} K+\widetilde{\Gamma}_{2}$. Since $\Gamma_{2}<\bar{\Gamma}_{2} K+\widetilde{\Gamma}_{2}$, then we can let $\varepsilon$ be sufficiently small such that $\Gamma_{2}-\bar{\Gamma}_{2} K-\widetilde{\Gamma}_{2}+2 \varepsilon<0$. Applying (i) in Lemma 6 to (37) results in $\lim _{t \rightarrow+\infty} N_{2}(t)=0$, a.s. The proof of $\lim _{t \rightarrow+\infty}\left\langle N_{1}(t)\right\rangle=$ $\eta_{1} / a_{11}$ a.s. is the same as that in (II) and hence is omitted. The proof of (B) is similar to that of (A) and hence is omitted.

(C) Assume that $\Gamma_{1}>\bar{\Gamma}_{1} K+\widetilde{\Gamma}_{1}$ and $\Gamma_{2}>\bar{\Gamma}_{2} K+\widetilde{\Gamma}_{2}$. Notice that $\Gamma_{2}>\widetilde{\Gamma}_{2}$; then, by (37) and Lemma 6,

$$
\limsup _{t \rightarrow+\infty}\left\langle N_{2}(t)\right\rangle \leq \frac{\Gamma_{2}-\bar{\Gamma}_{2} K-\widetilde{\Gamma}_{2}+2 \varepsilon}{\Gamma}, \quad \text { a.s. }
$$

It then follows from the arbitrariness of $\varepsilon$ that

$$
\limsup _{t \rightarrow+\infty}\left\langle N_{2}(t)\right\rangle \leq \frac{\Gamma_{2} \bar{\Gamma}_{2} K-\widetilde{\Gamma}_{2}}{\Gamma}, \quad \text { a.s. }
$$

Similarly, by (39), Lemma 6, and the arbitrariness of $\varepsilon$, we have

$$
\limsup _{t \rightarrow+\infty}\left\langle N_{1}(t)\right\rangle \leq \frac{\Gamma_{1}-\bar{\Gamma}_{1} K-\widetilde{\Gamma}_{1}}{\Gamma}, \quad \text { a.s. }
$$

Let $\varepsilon$ be sufficiently small satisfying $a_{11}\left(\left(\Gamma_{1}-\bar{\Gamma}_{1} K-\widetilde{\Gamma}_{1}\right) / \Gamma\right)-\varepsilon>$ 0 . Substituting (34), (41), and (20) into (22) yields

$$
\begin{aligned}
& t^{-1} \ln N_{1}(t) \\
& =t^{-1} \ln N_{1}(0)+r_{10}-r_{11}\left\langle C_{0}(t)\right\rangle-0.5 \lambda_{1}^{2} \\
& -a_{11}\left\langle N_{1}(t)\right\rangle-a_{12}\left\langle N_{2}(t)\right\rangle+\lambda_{1} W_{1} \frac{(t)}{t} \\
& +a_{12} t^{-1}\left[\int_{t-\tau_{1}}^{t} N_{2}(s) d s-\int_{-\tau_{1}}^{0} N_{2}(s) d s\right] \\
& \geq \eta_{1}-2 \varepsilon-a_{11}\left\langle N_{1}(t)\right\rangle-a_{12} \limsup _{t \rightarrow+\infty}\left\langle N_{2}(t)\right\rangle \\
& +\lambda_{1} W_{1} \frac{(t)}{t} \\
& \geq \eta_{1}-2 \varepsilon-a_{11}\left\langle N_{1}(t)\right\rangle-a_{12} \frac{\Gamma_{2}-\widetilde{\Gamma}_{2}}{\Gamma}+\lambda_{1} W_{1} \frac{(t)}{t} \\
& =a_{11} \frac{\Gamma_{1}-\bar{\Gamma}_{1} K-\widetilde{\Gamma}_{1}}{\Gamma}-2 \varepsilon-a_{11}\left\langle N_{1}(t)\right\rangle+\lambda_{1} W_{1} \frac{(t)}{t},
\end{aligned}
$$

for sufficiently large $t$. By (ii) in Lemma 6 and the arbitrariness of $\varepsilon$, one can observe that

$$
\liminf _{t \rightarrow+\infty}\left\langle N_{1}(t)\right\rangle \geq \frac{\Gamma_{1}-\bar{\Gamma}_{1} K-\widetilde{\Gamma}_{1}}{\Gamma}, \quad \text { a.s. }
$$

Similarly, when (34) and (42) and (20) are used in (9), we can see that $\liminf \inf _{t \rightarrow+\infty}\left\langle N_{2}(t)\right\rangle \geq\left(\Gamma_{2}-\bar{\Gamma}_{2} K-\widetilde{\Gamma}_{2}\right) / \Gamma$, a.s. This, together with (41), (42), and (44), indicates that

$$
\begin{aligned}
& \lim _{t \rightarrow+\infty}\left\langle N_{1}(t)\right\rangle=\frac{\Gamma_{1}-\bar{\Gamma}_{1} K-\widetilde{\Gamma}_{1}}{\Gamma}, \\
& \lim _{t \rightarrow+\infty}\left\langle N_{2}(t)\right\rangle=\frac{\Gamma_{2}-\bar{\Gamma}_{2} K-\widetilde{\Gamma}_{2}}{\Gamma}, \quad \text { a.s. }
\end{aligned}
$$




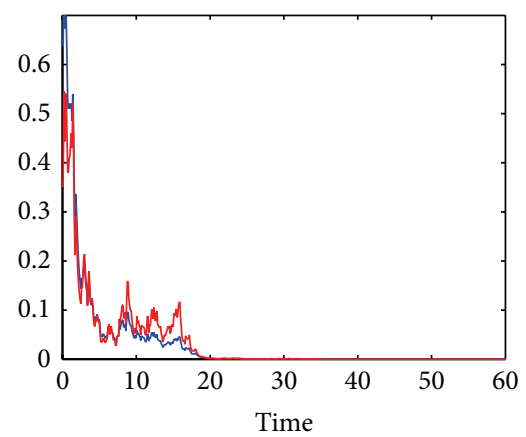

$-N_{1}$

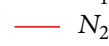

(a)

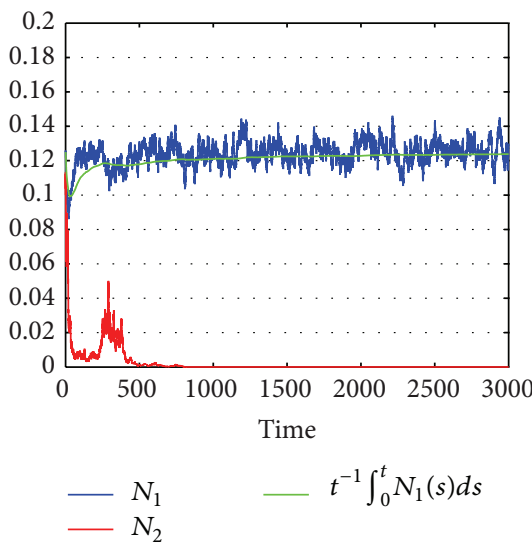

(d)

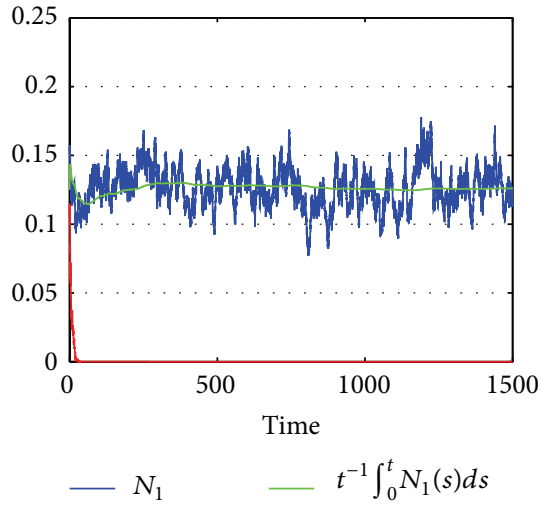

(b)

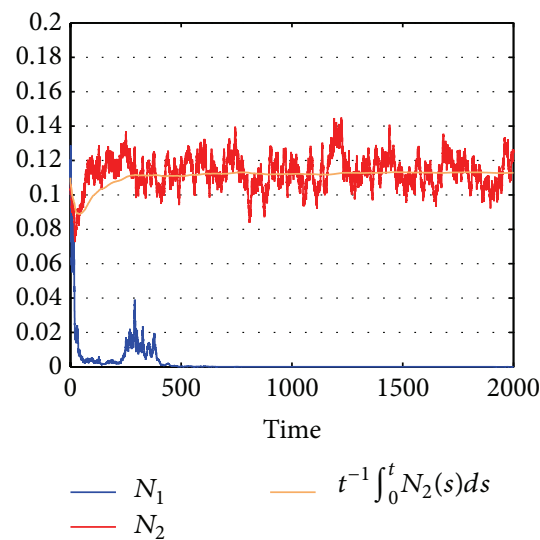

(e)

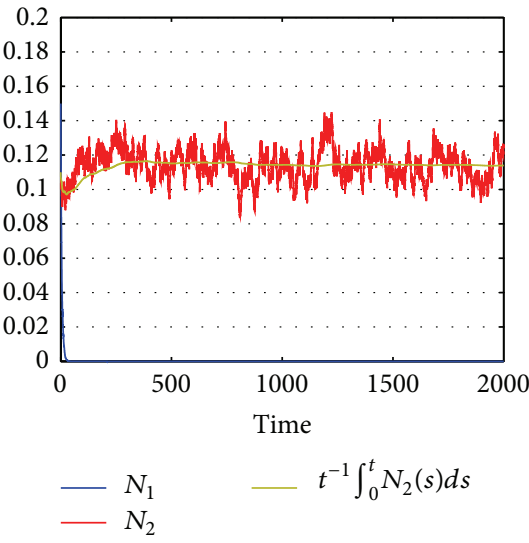

(c)

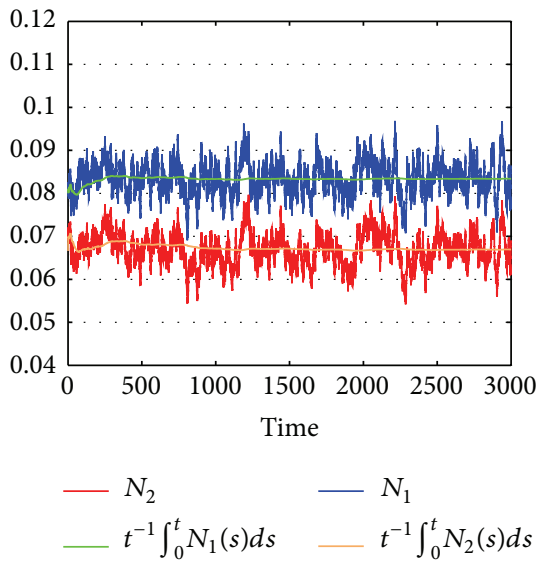

(f)

Figure 1: Solutions of system (1) for $r_{10}=0.7, r_{20}=0.5, a_{11}=0.8, a_{12}=0.5, a_{21}=0.4, a_{22}=0.7, \tau_{1}=10, \tau_{2}=12, k=g=m=0.2, h=0.5$, $b=0.1, \gamma=1, C_{0}(0)=C_{e}(0)=0.1$, and step size $\Delta t=0.001$. (a) is with $\lambda_{1}=1.1, \lambda_{2}=1$; (b) is with $\lambda_{1}=1, \lambda_{2}=1$; (c) is with $\lambda_{1}=1.2$, $\lambda_{2}=0.8$; (d) is with $\lambda_{1}=1, \lambda_{2}=0.8484$; (e) is with $\lambda_{1}=1.058, \lambda_{2}=0.8$; (f) is with $\lambda_{1}=1, \lambda_{2}=0.8$.

\section{Numerical Simulations}

In this section, using the classical Milstein method (see, e.g., [31]), we work out some numerical figures to support the analytical results. In Figure 1, we choose $r_{10}=0.7, r_{20}=0.5$, $a_{11}=0.8, a_{12}=0.5, a_{21}=0.4, a_{22}=0.7, \tau_{1}=10$, $\tau_{2}=12, k=g=m=0.2, h=0.5, b=0.1$, and $\gamma=1$. Then, $\Gamma=0.36, \Gamma_{1}=0.24, \Gamma_{2}=0.12, \overline{\Gamma_{1}}=0.2, \overline{\Gamma_{2}}=0.4$, and $K=k b / \gamma h(g+m)=0.1$. The only difference between conditions of Figures 1(a), 1(b), 1(c), 1(d), 1(e), and 1(f) is that the values of $\lambda_{1}$ and $\lambda_{2}$ are different.

(a) In Figure 1(a), we choose $\lambda_{1}=1.1, \lambda_{2}=1$. Then, $\eta_{1}=r_{10}-r_{11} K-0.5 \lambda_{1}^{2}=-0.12, \eta_{2}=r_{20}-r_{21} K-$ $0.5 \lambda_{2}^{2}=-0.1$. By virtue of (I) in Theorem 2, both $N_{1}$ and $\mathrm{N}_{2}$ are extinct; see Figure 1(a).

(b) In Figure $1(\mathrm{~b})$, we set $\lambda_{1}=1, \lambda_{2}=1$. Then, $\eta_{1}=$ $0.1, \eta_{2}=-0.1$. In view of (II) in Theorem $2, N_{2}$ is extinct and $\lim _{t \rightarrow+\infty} t^{-1} \int_{0}^{t} N_{1}(s) d s=\eta_{1} / a_{11}=0.125$. Figure 1(b) confirms this. (c) In Figure $1(\mathrm{c})$, we let $\lambda_{1}=1.2, \lambda_{2}=0.8$. Then, $\eta_{1}=$ $-0.12, \eta_{2}=0.08$. It follows from (III) in Theorem 2 that $N_{1}$ is extinct and

$$
\lim _{t \rightarrow+\infty} t^{-1} \int_{0}^{t} N_{2}(s) d s=\frac{\eta_{2}}{a_{22}}=0.1143 .
$$

See Figure 1(c).

(d) In Figure $1(\mathrm{~d})$, we set $\lambda_{1}=1, \lambda_{2}=0.8484$. Then, $\eta_{1}=$ $0.1, \eta_{2}=0.04, \Gamma_{1}=0.24>\bar{\Gamma}_{1} K+\widetilde{\Gamma}_{1}=0.19$, and $\Gamma_{2}=0.12<\bar{\Gamma}_{2} K+\widetilde{\Gamma}_{2}=0.128$. According to (A) in Theorem $2, N_{2}$ is extinct and

$$
\lim _{t \rightarrow+\infty} t^{-1} \int_{0}^{t} N_{1}(s) d s=\frac{\eta_{1}}{a_{11}}=\frac{0.1}{0.8}=0.125 .
$$

Figure $1(\mathrm{~d})$ confirms this.

(e) In Figure 1(e), we choose $\lambda_{1}=1.058, \lambda_{2}=0.8$. Then, $\eta_{1}=0.04, \eta_{2}=0.08, \Gamma_{1}=0.24<\bar{\Gamma}_{1} K+\widetilde{\Gamma}_{1}=0.252$, and 
$\Gamma_{2}=0.12>\bar{\Gamma}_{2} K+\widetilde{\Gamma}_{2}=0.072$. By (B) in Theorem 2, $N_{1}$ is extinct and

$$
\lim _{t \rightarrow+\infty} t^{-1} \int_{0}^{t} N_{2}(s) d s=\frac{\eta_{2}}{a_{22}}=\frac{0.08}{0.7}=0.1143
$$

See Figure 1(e).

(f) In Figure 1(f), we let $\lambda_{1}=1, \lambda_{2}=0.8$. Then, $\Gamma_{1}=$ $0.24>\bar{\Gamma}_{1} K+\widetilde{\Gamma}_{1}=0.21$ and $\Gamma_{2}=0.12>\bar{\Gamma}_{2} K+\widetilde{\Gamma}_{2}=$ 0.096. It follows from (C) in Theorem 2 that

$$
\begin{aligned}
& \lim _{t \rightarrow+\infty} t^{-1} \int_{0}^{t} N_{1}(s) d s=\frac{\Gamma_{1}-\bar{\Gamma}_{1} K-\widetilde{\Gamma}_{1}}{\Gamma}=\frac{0.03}{0.36}=0.0833, \\
& \lim _{t \rightarrow+\infty} t^{-1} \int_{0}^{t} N_{2}(s) d s=\frac{\Gamma_{2}-\bar{\Gamma}_{2} K-\widetilde{\Gamma}_{2}}{\Gamma}=0.0667
\end{aligned}
$$

See Figure 1(f).

\section{Conclusions and Future Directions}

This paper investigates a stochastic delay competitive model in a polluted environment with impulsive toxicant input. For each population, the critical value between stability in the mean and extinction is obtained. Some recent works are extended and improved. Our Theorem 2 has some important and interesting interpretation.

(1) Time delay is harmless for stability in the mean and extinction of the stochastic model (1).

(2) White noises can change the dynamics of the population model greatly.

Some interesting problems deserve further study. One can consider some more realistic systems, for example, stochastic delayed population model with the Markov switching (see, e.g., $[22,23,29])$. It is also interesting to extend Theorem 2 to $n$-species case.

\section{Conflict of Interests}

The authors declare that they have no conflict of interests regarding the publication of this paper.

\section{Acknowledgments}

The authors thank the reviewers for their valuable comments. The authors also thank Dr. H. Qiu and Dr. C. Zhang for helping them to improve the English exposition. This research is supported by National Natural Science Foundation of PR China (nos. 11301207, 11171081, 11271364, and 11301112), Natural Science Foundation of Jiangsu Province (nos. BK2011407 and BK20130411), and Natural Science Research Project of Ordinary Universities in Jiangsu Province (no. 13KJB110002).

\section{References}

[1] T. G. Hallam, C. E. Clark, and R. R. Lassiter, "Effects of toxicants on populations: a qualitative approach. 1. Equilibrium environmental exposure," Ecological Modelling, vol. 18, no. 4, pp. 291-304, 1983.

[2] T. G. Hallam, C. E. Clark, and G. S. Jordan, "Effects of toxicants on populations: a qualitative approach. II. First order kinetics," Journal of Mathematical Biology, vol. 18, no. 1, pp. 25-37, 1983.

[3] T. G. Hallam and J. T. de Luna, "Effects of toxicants on populations: a qualitative approach. III. Environmental and food chain pathways," Journal of Theoretical Biology, vol. 109, no. 3, pp. 411-429, 1984.

[4] Z. E. Ma, G. R. Cui, and W. D. Wang, "Persistence and extinction of a population in a polluted environment," Mathematical Biosciences, vol. 101, no. 1, pp. 75-97, 1990.

[5] H. I. Freedman and J. B. Shukla, "Models for the effect of toxicant in single-species and predator-prey systems," Journal of Mathematical Biology, vol. 30, no. 1, pp. 15-30, 1991.

[6] H. Liu and Z. Ma, "The threshold of survival for system of two species in a polluted environment," Journal of Mathematical Biology, vol. 30, no. 1, pp. 49-61, 1991.

[7] Z. Ma, W. Zong, and Z. Luo, "The thresholds of survival for an $n$-dimensional food chain model in a polluted environment," Journal of Mathematical Analysis and Applications, vol. 210, no. 2, pp. 440-458, 1997.

[8] B. Buonomo, A. di Liddo, and I. Sgura, "A diffusive-convective model for the dynamics of population-toxicant interactions: some analytical and numerical results," Mathematical Biosciences, vol. 157, no. 1-2, pp. 37-64, 1999.

[9] J. Pan, Z. Jin, and Z. Ma, “Thresholds of survival for an $n$ dimensional Volterra mutualistic system in a polluted environment," Journal of Mathematical Analysis and Applications, vol. 252, no. 2, pp. 519-531, 2000.

[10] J. He and K. Wang, "The survival analysis for a population in a polluted environment," Nonlinear Analysis: Real World Applications, vol. 10, no. 3, pp. 1555-1571, 2009.

[11] S. Sinha, O. P. Misra, and J. Dhar, "Modelling a predator-prey system with infected prey in polluted environment," Applied Mathematical Modelling, vol. 34, no. 7, pp. 1861-1872, 2010.

[12] B. Liu, L. Chen, and Y. Zhang, "The effects of impulsive toxicant input on a population in a polluted environment," Journal of Biological Systems, vol. 11, no. 3, pp. 265-274, 2003.

[13] X. Yang, Z. Jin, and Y. Xue, "Weak average persistence and extinction of a predator-prey system in a polluted environment with impulsive toxicant input," Chaos, Solitons \& Fractals, vol. 31, no. 3, pp. 726-735, 2007.

[14] F. Tao and B. Liu, "Dynamic behaviors of a single-species population model with birth pulses in a polluted environment," The Rocky Mountain Journal of Mathematics, vol. 38, no. 5, pp. 1663-1684, 2008.

[15] B. Liu and L. Zhang, "Dynamics of a two-species LotkaVolterra competition system in a polluted environment with pulse toxicant input," Applied Mathematics and Computation, vol. 214, no. 1, pp. 155-162, 2009.

[16] J. Jiao, W. Long, and L. Chen, "A single stage-structured population model with mature individuals in a polluted environment and pulse input of environmental toxin," Nonlinear Analysis: Real World Applications, vol. 10, no. 5, pp. 3073-3081, 2009.

[17] X. Meng, Z. Li, and J. J. Nieto, "Dynamic analysis of MichaelisMenten chemostat-type competition models with time delay and pulse in a polluted environment," Journal of Mathematical Chemistry, vol. 47, no. 1, pp. 123-144, 2010.

[18] J. Jiao, S. Cai, and L. Chen, "Dynamics of the genic mutational rate on a population system with birth pulse and impulsive 
input toxins in polluted environment," Journal of Applied Mathematics and Computing, vol. 40, no. 1-2, pp. 445-457, 2012.

[19] R. M. May, Stability and Complexity in Model Ecosystems, Princeton University Press, Princeton, NJ, USA, 2001.

[20] K. Gopalsamy, Stability and Oscillations in Delay Differential Equations of Population Dynamics, vol. 74 of Mathematics and Its Applications, Kluwer Academic Publishers, Dordrecht, The Netherlands, 1992.

[21] M. Liu and K. Wang, "Dynamics of a Leslie-Gower Holling-type II predator-prey system with Lévy jumps," Nonlinear Analysis: Theory, Methods \& Applications, vol. 85, pp. 204-213, 2013.

[22] M. Liu and K. Wang, "Stochastic Lotka-Volterra systems with Lévy noise," Journal of Mathematical Analysis and Applications, vol. 410, no. 2, pp. 750-763, 2014.

[23] X. Li and X. Mao, "Population dynamical behavior of nonautonomous Lotka-Volterra competitive system with random perturbation," Discrete and Continuous Dynamical Systems, vol. 24, no. 2, pp. 523-545, 2009.

[24] M. Liu, K. Wang, and Q. Wu, "Survival analysis of stochastic competitive models in a polluted environment and stochastic competitive exclusion principle," Bulletin of Mathematical Biology, vol. 73, no. 9, pp. 1969-2012, 2011.

[25] M. Liu and K. Wang, "Stochastic logistic equation with infinite delay," Mathematical Methods in the Applied Sciences, vol. 35, no. 7, pp. 812-827, 2012.

[26] M. Liu and K. Wang, "A note on a delay Lotka-Volterra competitive system with random perturbations," Applied Mathematics Letters, vol. 26, no. 6, pp. 589-594, 2013.

[27] M. Liu and K. Wang, "Population dynamical behavior of LotkaVolterra cooperative systems with random perturbations," Discrete and Continuous Dynamical Systems, vol. 33, no. 6, pp. 2495-2522, 2013.

[28] M. Liu and K. Wang, "Analysis of a stochastic autonomous mutualism model," Journal of Mathematical Analysis and Applications, vol. 402, no. 1, pp. 392-403, 2013.

[29] Y. Liu, Q. Liu, and Z. Liu, "Dynamical behaviors of a stochastic delay logistic system with impulsive toxicant input in a polluted environment," Journal of Theoretical Biology, vol. 329, pp. 1-5, 2013.

[30] N. Ikeda and S. Watanabe, "A comparison theorem for solutions of stochastic differential equations and its applications," Osaka Journal of Mathematics, vol. 14, no. 3, pp. 619-633, 1977.

[31] P. E. Kloeden and T. Shardlow, "The Milstein scheme for stochastic delay differential equations without using anticipative calculus," Stochastic Analysis and Applications, vol. 30, no. 2, pp. 181-202, 2012. 


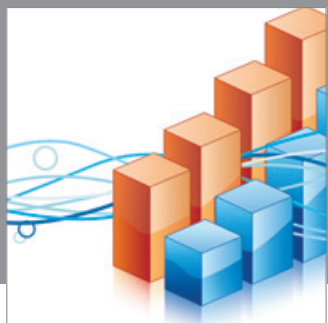

Advances in

Operations Research

mansans

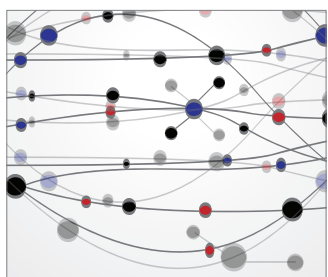

The Scientific World Journal
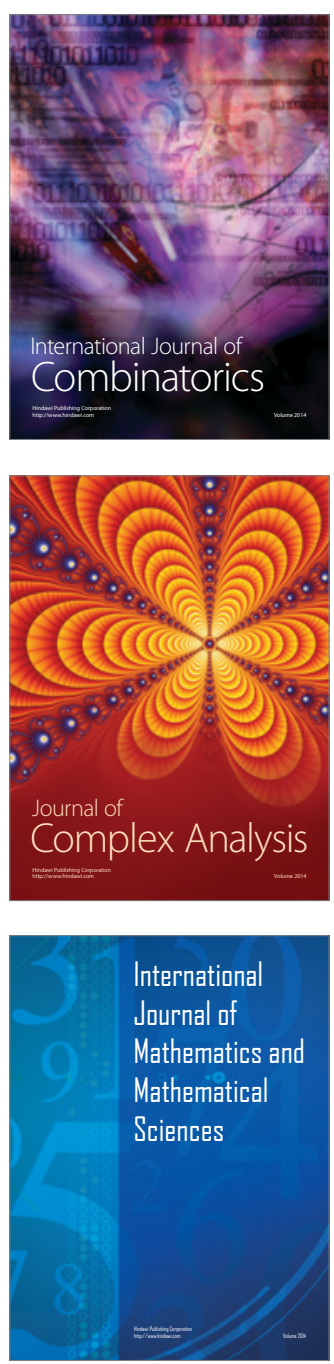
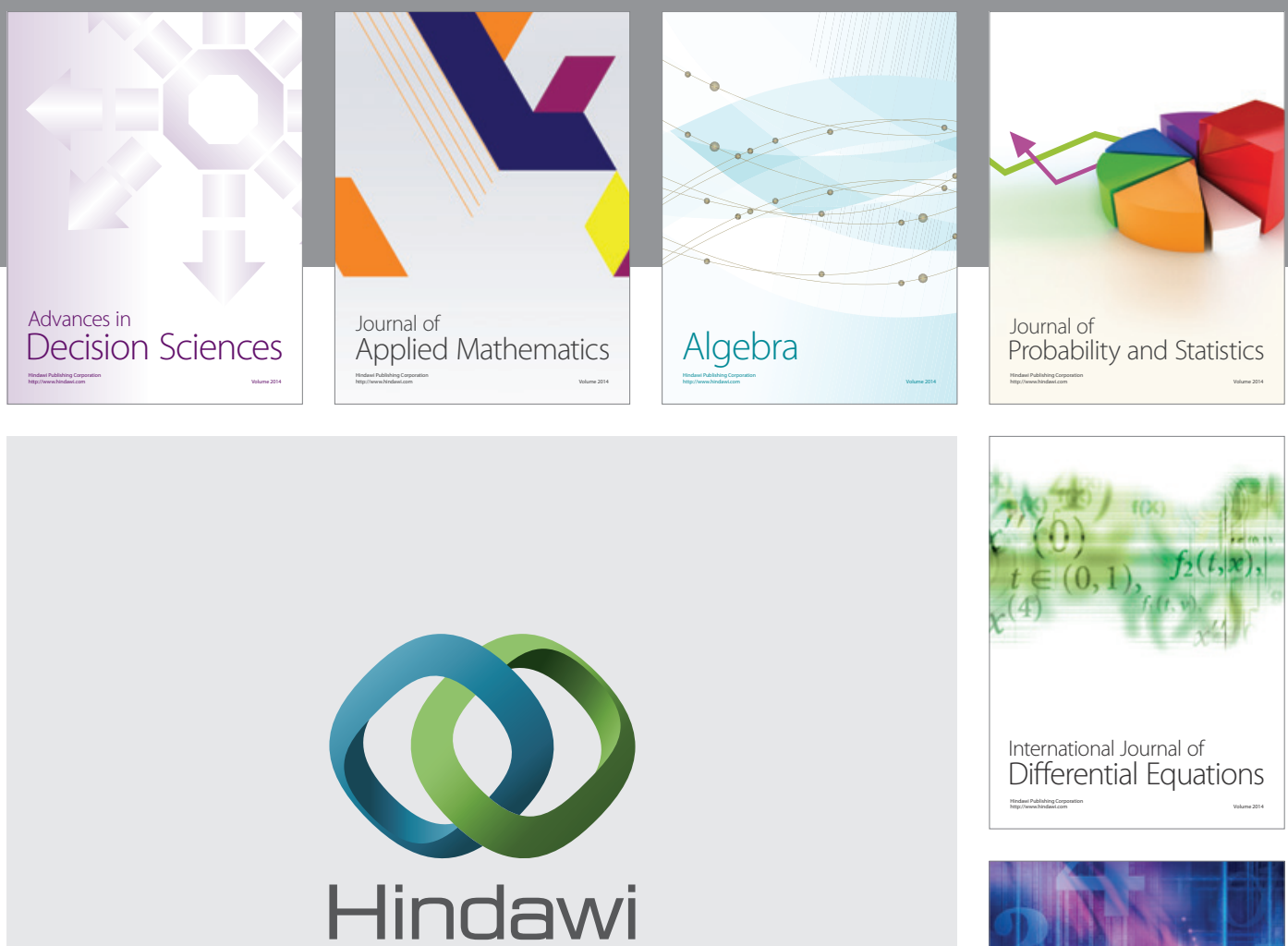

Submit your manuscripts at http://www.hindawi.com
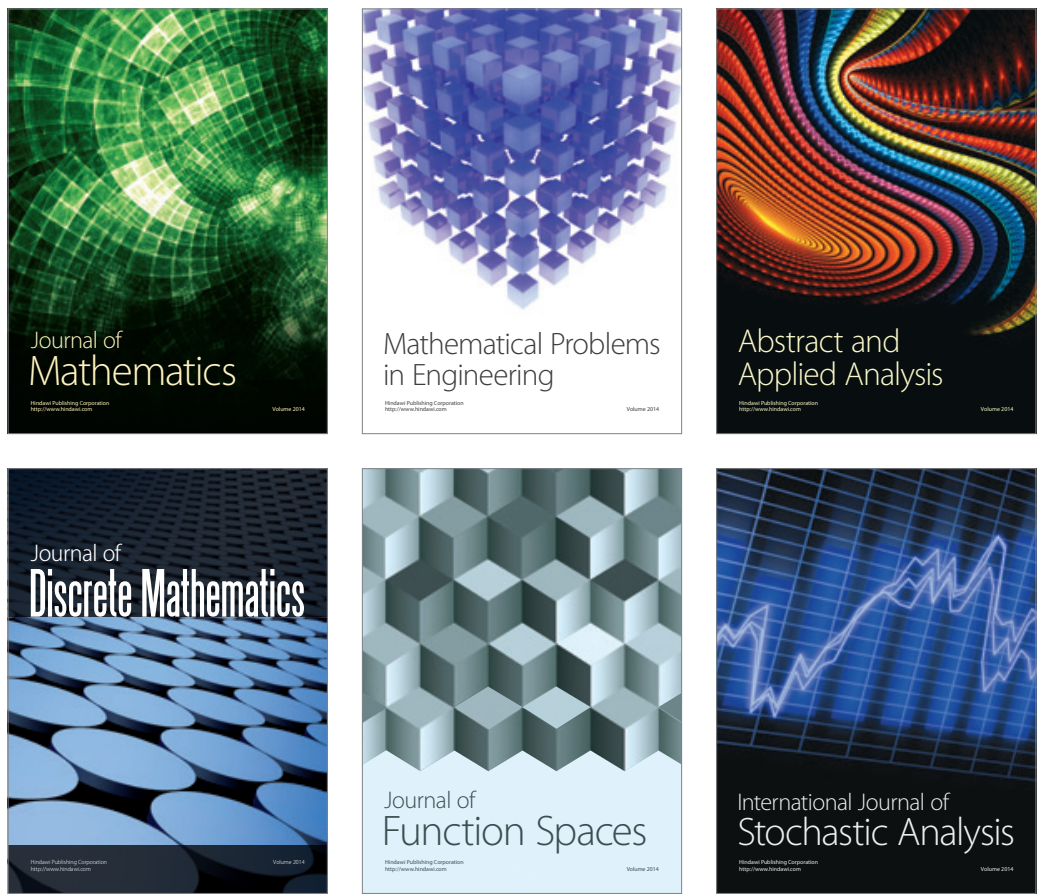

Journal of

Function Spaces

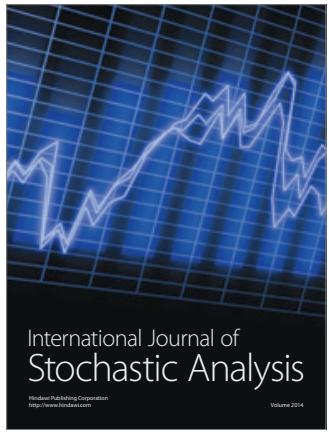

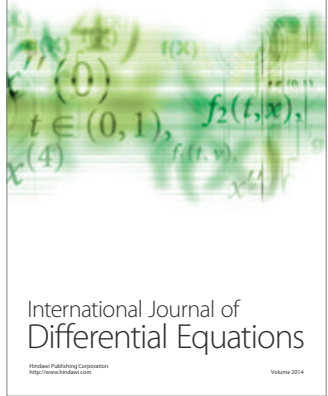
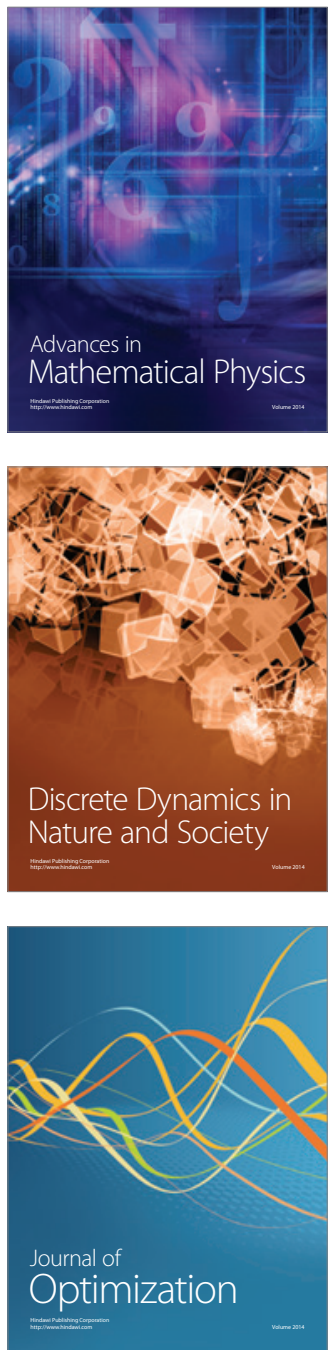